\title{
Article \\ Relationship between Anti-Wrinkle Property of Cotton Fabrics and Crosslinking Properties of Glycosyl Polyaldehydes and Polyuronic Acids Finishing Agents: A Molecular Simulation Study
}

\author{
Jiangfei Lou, Dan Wang, Jiugang Yuan and Xuerong Fan *
}

check for

updates

Citation: Lou, J.; Wang, D.; Yuan, J.; Fan, X. Relationship between Anti-Wrinkle Property of Cotton Fabrics and Crosslinking Properties of Glycosyl Polyaldehydes and Polyuronic Acids Finishing Agents: A Molecular Simulation Study. Appl. Sci. 2021, 11, 10994. https://doi.org/ 10.3390/app112210994

Academic Editor: Cezary Czaplewski

Received: 28 September 2021

Accepted: 17 November 2021

Published: 19 November 2021

Publisher's Note: MDPI stays neutral with regard to jurisdictional claims in published maps and institutional affiliations.

Copyright: (c) 2021 by the authors. Licensee MDPI, Basel, Switzerland. This article is an open access article distributed under the terms and conditions of the Creative Commons Attribution (CC BY) license (https:/ / creativecommons.org/licenses/by/ $4.0 /)$.
Key Laboratory of Science and Technology of Eco-Textile, Ministry of Education, Jiangnan University, Wuxi 214122, China; jiangfeilou@stu.jiangnan.edu.cn (J.L.); 7190707009@stu.jiangnan.edu.cn (D.W.); jiugangyuan@jiangnan.edu.cn (J.Y.)

* Correspondence: 8093200024@jiangnan.edu.cn

\begin{abstract}
In this paper, monosaccharide (glucose and fructose), disaccharide (sucrose and trehalose), trisaccharide (raffinose) and tetrasaccharide (stachyose) were selected as the research objects and the glycosyl polyaldehydes and glycosyl polyuronic acid anti-wrinkle finishing agents were prepared via selective oxidation. The anti-wrinkle properties of their finished fabrics were analyzed, the molecular radius, the number of reactive groups, the number of reaction sites and the number of rotatable bonds of the finishing agent were calculated to evaluate its diffusion rate and crosslinking characteristics inside the cellulose. Through molecular simulation, the number of possible conformations of the anti-wrinkle finishing agent with the single cross-linked state was calculated, and the distance between different cross-linking points was measured, and the relationship between the effective cross-linking radius and the anti-wrinkle performance was studied. The results showed that trehalose polyaldehydes, raffinose polyaldehydes, trehalose polyuronic acid, and raffinose polyuronic acid finished fabrics had an excellent anti-wrinkle property, the strength retention rates of the fabrics were all above $68 \%$, and the whiteness index was above 70 . The smaller the molecular radius was, the easier the finishing agent was to diffuse into the cellulose. The most suitable crosslinking radius of glycosyl finishing agent was 3.5-6.0 Å.
\end{abstract}

Keywords: glycosyl finishing agents; anti-wrinkle finishing; crosslinking property; molecular volume; molecular simulation; effective cross-linking range

\section{Introduction}

Cotton fabrics are favored by people for their excellent wearing comfort and breathability. However, cotton fabrics can cause many inconveniences to people due to wrinkles in washing and daily wear. With the increasing demand for natural fibers, anti-wrinkle finishing has become an important post-finishing process in the dyeing and finishing of cotton fabric [1,2]. Anti-wrinkle finishing agents for cotton fabrics can be divided into the following two categories: formaldehyde finishing agents and formaldehyde-free finishing agents. Formaldehyde finishing agents are mainly N-methylol resin compounds, such as dimethylol dihydroxy ethylene urea (DMDHEU, 2D resin), this type of compound has high reactivity and an excellent cross-linking effect, but the ether bond is easy to hydrolyze and break and releases free formaldehyde, which is harmful to human health $[3,4]$. As an alternative finishing agent for 2D resin products, formaldehyde-free finishing agents mainly include dialdehydes, epoxy resins, polyurethanes, silicones, polycarboxylic acids and ionomers. The cotton fabrics with these finishing agents have an excellent wrinkle property, but there are problems such as strong damage to and yellowing of the fabric [5-7].

The anti-wrinkle finishing of the cotton fabric chemical cross-linking method generally requires that the cross-linking agent has a suitable molecular size, which can enter the fiber 
and form cross-links with cellulose on the inside and surface of the fiber. The anti-wrinkle finishing process is similar to the dyeing process of reactive dyes on cotton fabrics (as shown in Figure 1) [4,8]. The specific process is after the cotton fabric is dipped in the anti-wrinkle finishing solution, the crosslinking agent molecules diffuse to the fiber surface boundary layer and adsorb on the surface of the cotton fiber; then, diffusion into the fiber, a cross-linking reaction with cellulose, and fixation within the fiber takes place.

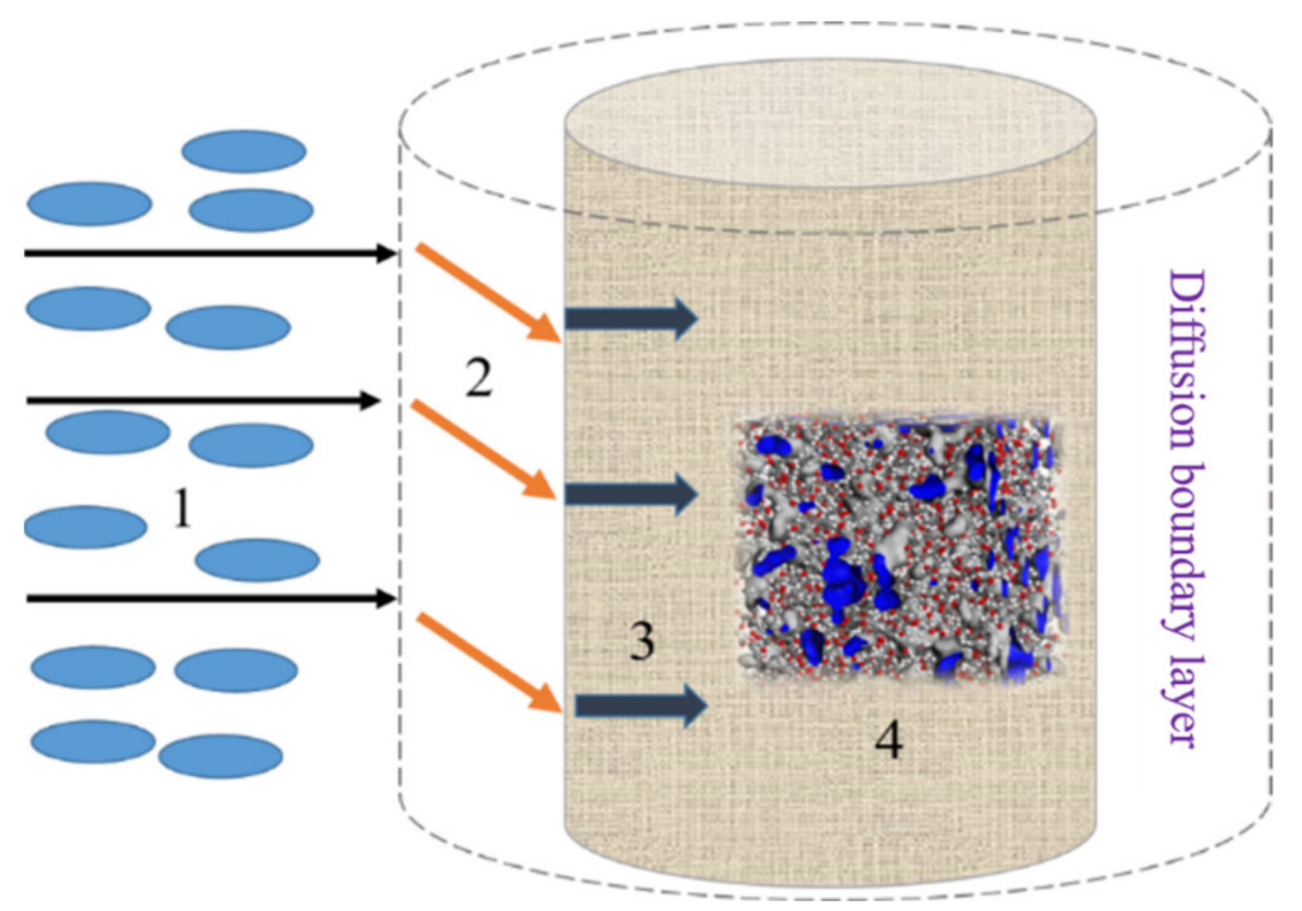

Figure 1. Molecular diffusion model. 1. Diffusing to the fiber surface boundary layer; 2 . Being absorbed by the surface of cotton fiber; 3 . Diffusing into the fiber; 4 . Cross-linking with cellulose and fixing in the fiber.

At present, the development and research of formaldehyde-free anti-wrinkle finishing of cotton fabrics has entered a bottleneck period, and it mainly focuses on the crosslinking mechanism of polycarboxylic acid finishing agents, the finishing process, the influence of additives, and the compound application of polycarboxylic acids $[2,6]$. Few researchers have designed and prepared a new type of anti-wrinkle finishing agent starting from the type, number, and reactivity of the crosslinking agent reactive groups. This article took monosaccharides (glucose and fructose), disaccharides (sucrose and trehalose), trisaccharides (raffinose) and tetrasaccharides (stachyose) as the research objects to prepare corresponding glycosyl formaldehyde-free anti-wrinkle finishing agents (the process of preparation is shown in Figure 2). It was also used in the anti-wrinkle finishing of cotton fabrics to evaluate the difference in anti-wrinkle performance. We used Chem3D 19.0 software to calculate the molecular volume of the finishing agent and evaluate its diffusion rate in the fiber; calculated the number of reactive groups, the number of reaction sites and the number of rotatable bonds of the finishing agents; and analyzed the crosslinking properties of different finishing agents. We performed a molecular dynamics simulation with gromacs software, calculated the number of conformations and the conformational energy that the finishing agent existed in within a single cross-linked state, measured the distance between the different cross-linking points, and revealed the relationship between the effective cross-linking range and the cross-linking property of different finishing agents. 


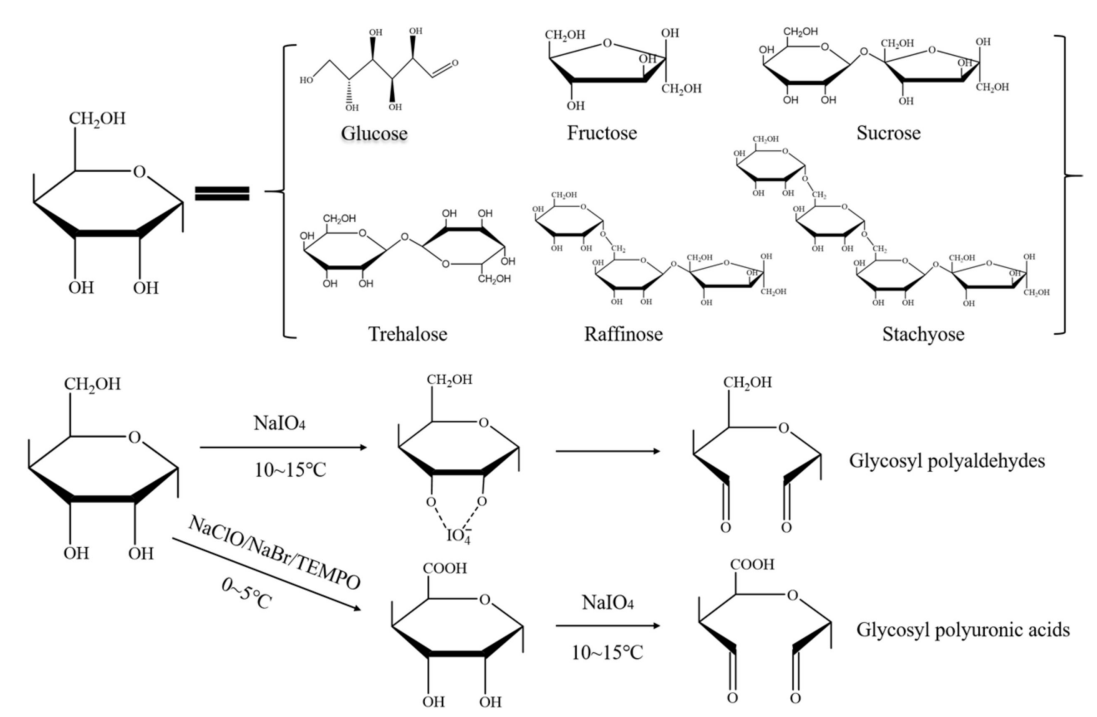

Figure 2. Schematic of preparation mechanism of glycosyl anti-wrinkle finishing agents.

\section{Experimental}

\subsection{Materials}

Unbleached plain cotton fabric ( $100 \%$, yarn count: 10 tex $\times 10$ tex, warp and weft density/inch: 606 pieces $\times 318$ pieces, weight: $113 \mathrm{~g} / \mathrm{m}^{2}$, width: $1.50 \mathrm{~m}$ ) was purchased from the supplier (Shandong Ruyi Group Co., Ltd., Jining, China). Glucose, fructose, sucrose, trehalose, raffinose and stachyose, magnesium chloride, sodium hypophosphite monohydrate, sodium bromide, sodium hypochlorite, and sodium hydroxide were purchased from Sinopharm Chemical Reagent Co., Ltd. Sodium periodate, 2,2,6,6-tetramethylpiperidine oxide (TEMPO) was purchased from Shanghai Mclean biochemical Technology Co., Ltd. Three crosslinking agents, glutaraldehyde (25\% solution, Sinopharm Chemical Reagent Co., Ltd., Shanghai, China), DMDHEU (Dymatic Chemical Reagent Co., Ltd., Guangzhou, China), and BTCA (J\&K Scientific Ltd., Beijing, China), were used. All the chemicals were of reagent grade and used without further purification.

\subsection{Methods}

\subsubsection{Preparation of the Glycosyl Polyaldehydes}

The preparation of glycosyl polyaldehydes was used in the selective oxidation system of sodium periodate, which was carried out according to the procedure with a slight modification [9-11]. In brief, fructose, sucrose, trehalose, raffinose, and stachyose $(0.03 \mathrm{~mol})$ were dissolved in $300 \mathrm{~mL}$ of water and $\mathrm{NaIO}_{4}(0.15 \mathrm{~mol})$ was added. After the reaction mixture was stirred in the dark at $20^{\circ} \mathrm{C}$ for $24 \mathrm{~h}$, barium dichloride $(0.08 \mathrm{~mol})$ was added and stirred at $10^{\circ} \mathrm{C}$ for $24 \mathrm{~h}$. The mixture was filtered, and the solution containing the polyaldehyde derivative was prepared. Then, the solution of polyaldehyde derivative was kept at $-20{ }^{\circ} \mathrm{C}$ and dried by lyophilization to obtain glycosyl polyaldehydes powder.

\subsubsection{Preparation of the Glycosyl Polyuronic Acid}

The preparation of glycosyl polyuronic acid required two steps of selective oxidation. The first step modified the TEMPO-mediated oxidation of cellulose, as reported previously [12]. Briefly, fructose, sucrose, trehalose, raffinose, and stachyose $(4 \mathrm{mmol})$ were dissolved in water $(200 \mathrm{~mL})$, which containing TEMPO and sodium bromide. A NaClO solution ( $\mathrm{pH}$ was adjusted to 10.5 ) was added to the reaction solution at $0-5{ }^{\circ} \mathrm{C}$, and the $\mathrm{pH}$ was maintained at 10.5 with a $0.5 \mathrm{~mol} / \mathrm{L}$ sodium hydroxide solution; then, the $\mathrm{pH}$ did not change in the $30 \mathrm{~min}$ after the addition; therefore, we stopped the reaction with $95 \%$ ethanol, and then adjusted to 7.0 with $0.5 \mathrm{~mol} / \mathrm{L} \mathrm{HCl}$. The glycosyl carboxylic acid was kept at $-20^{\circ} \mathrm{C}$, then dried by lyophilization to obtain the powder. 
The second step used the method of Section 2.2.1; the glycosyl carboxylic acid prepared in the first step was selectively oxidized by $\mathrm{NaIO}_{4}$ to prepare glycosyl polyuronic acid.

\subsubsection{Anti-Crease Treatment of Cotton Fabric}

The fabric sample was first immersed in an aqueous solution containing either glycosyl polyaldehydes or glycosyl polyuronic acid or DMDHEU, or glutaraldehyde, or BTCA with some additives, then padded using a two-bowl horizontal laboratory padder (Yalinuo, Guangzhou, China) to attain a wet pick-up of $80-90 \%$. Impregnated fabrics were dried and then cured at the specified condition in the curing condition (the curing condition of glycosyl polyaldehydes, DMDHEU, and glutaraldehyde was $3 \mathrm{~min}$ at $80^{\circ} \mathrm{C}$ and $3 \mathrm{~min}$ at $160^{\circ} \mathrm{C}$; the curing condition of glycosyl polyuronic acid was $3 \mathrm{~min}$ at $80^{\circ} \mathrm{C}, 3 \mathrm{~min}$ at $160{ }^{\circ} \mathrm{C}$ and $2 \mathrm{~min}$ at $180^{\circ} \mathrm{C}$; the curing condition of BTCA was $3 \mathrm{~min}$ at $80^{\circ} \mathrm{C}$ and $2 \mathrm{~min}$ at $180^{\circ} \mathrm{C}$ ) manufactured by Rapid Precion Machinery Co. Ltd., Xiamen, China.

\subsubsection{Properties of Treated Fabrics}

The wrinkle recovery angle (WRA) of the treated fabrics was measured according to the AATCC Test Method 66-2003. Each sample was measured 5 times in the weft (f) and warp $(\mathrm{w})$, and the average value of each group was taken.

The whiteness index (WI) of the treated fabrics were determined using the AATCC Test Method 110-2005. The Datacolor $650^{\circledR}$ Bench top spectrophotometer was used to test four different positions, and the average of every sample was calculated.

The tensile strength (TS) of the treated fabrics was determined with the ASTM Testing Method D-1424-1996; the test method was as follows: we took 5 groups of $5 \mathrm{~cm} \times 25 \mathrm{~cm}$ fabric strips along the warp and weft directions, set the clamping distance of the fabric strength tester to $20 \mathrm{~cm}$ using the HD026NS "Fabric strength tester" (Nantong Hongda Experimental Instrument Co., Ltd., Jiangsu China), recorded the test results, and calculated the average value using Equation (1), which is as follows:

$$
T S=T_{t} / T_{u} * 100 \%
$$

where $T_{\mathrm{t}}$ is the tensile strength $(\mathrm{cN})$ of the treated fabric and $T_{\mathrm{u}}$ is the tensile strength $(\mathrm{cN})$ of the untreated fabric.

All mechanical properties were measured 5 times in the warp and weft directions.

\subsubsection{Molecular Volume (CSEV)}

We used ChemDraw 19.0 to draw the molecular structure of the glycosyl anti-wrinkle finishing agents, BTCA, GA, and DMDHEU, and then calculated CSEV using the MM2 method of molecular mechanics in Chem3D 19.0. A simple dynamic simulation of the molecular configuration of the finishing agent can also be performed [13,14]. In the software Chem3D 19.0, the molecular structure of the finishing agent was optimized by the MM2 molecular mechanics configuration to obtain the minimum energy conformation. The Conley Discharge Volume (CSEV) considered the molecule of the finishing agent as the spherical model, which can reflect the size of the molecule.

\subsubsection{Number of Rotatable Bonds (RBN)}

According to the characteristics and binding mechanism of each of the finishing agents, the number of Potential Cross-linkable Groups (PCG) and the number of reaction sites (NRS) that can undergo a cross-linking reaction was analyzed. We calculated the number of rotatable bonds (RBN) of each finishing agent through Chem3D 19.0 to evaluate the possibility of rotating the finishing agent molecules and the number of different conformations. The RBN value also indicated the flexibility of the finishing agents; the larger the RBN value is, the greater the possibility that the molecule can be rotated, and the easier it is for the molecule to rotate to form more conformations. 
2.2.7. Calculation of Molecular Cross-Linking Distribution Range of Anti-Wrinkle Finishing Agent

There are free-rotating bonds in the compound molecule; a compound molecule contains many different molecular conformations. Although these conformations have different energies, the probability of their energy distribution and the proportion of the conformation conforms to the Boltzmann equilibrium distribution; therefore, the Boltzmann formula can be used to estimate energy and proportion as in the following Equation (2):

$$
P_{i}=\frac{\exp \left(-E_{i} / k T\right)}{\sum_{j=1}^{M} \exp \left(-E_{j} / k T\right)}
$$

where $P_{i}$ is the proportion of energy in the conformation of molecule $i ; E_{i}$ is the total energy in the conformation of molecule $i ; k$ is the Boltzmann formula constant; $T$ is the thermodynamic temperature $(\mathrm{K}), M$ is the total number of all conformations of the molecule; and $E j$ is the total energy of the molecular conformation in the $j$ molecular conformation.

Assuming that one end of the finishing agent was cross-linked with cellulose, the molecular dynamics simulation of 100,000 fs was carried out through gromacs software, and the simulation temperature was $600 \mathrm{~K}$. The structures were collected every $50 \mathrm{fs}$, and a total of 2000 structures were obtained, and these 2000 structures were optimized using the GFN1-xT method [15-18]. Through the clustering method, structures with a similar energy and similar structure will be classified as one structure, and appropriate energy and structure thresholds were selected, and each molecule has 15-20 structures after clustering. Then, took the 10 structures with the lowest energy for each clustered structure to perform a density functional theory (DFT) calculation, and calculated the energy probability distribution of the crosslinking agent under different crosslinking conditions.

The distance distribution from the reaction site to the fixed end under the corresponding cross-linking was measured using Chem-craft software, and the relationship between the effective cross-linking range of different finishing agents and the cross-linking effect was evaluated $[19,20]$.

\section{Results and Discussion}

\subsection{Anti-Wrinkle Performance with Glycosyl Formaldehyde-Free Finishing Agents}

Before discussing the molecular structure and properties of glycosyl formaldehydefree finishing agents, it was necessary to analyze the anti-wrinkle property of cotton fabrics with those finishing agents. The structure of glycosyl polyaldehydes finishing agents $(\mathrm{OFr}$, $\mathrm{OSu}, \mathrm{OTr}, \mathrm{ORa}, \mathrm{OSt}$ ) and the glycosyl polyuronic acid finishing agents (openSu, openTr, openRa, openSt) were shown in Figure 3. As monosaccharides will only produce one carboxyl group after carboxylation, a single carboxyl group cannot be cross-linked with hydroxyl groups according to the esterification cross-linking mechanism of polycarboxylic acid finishing agents; therefore, monosaccharides did not undergo a two-step oxidation reaction. Of these finished cotton fabrics with these glycosyl finishing agents and nonfinishing cotton fabrics, the BTCA, GA, and DMDHEU finished fabrics were set as the control samples, and the wrinkle recovery angle (WRA), whiteness index (WI), and tensile strength (TS) of the finished fabric were measured, and the results are shown in Figure 4.

From Figure 4, the anti-wrinkle performance of fabrics finished with glucose and the OFr finishing agent failed to reach the standard range of cotton fabric anti-wrinkle performance $\left(\mathrm{WRA} \geq 250^{\circ}\right)[1,3]$. The anti-wrinkle performance of the fabrics finished with disaccharides, trisaccharides, and tetrasaccharides glycosyl anti-wrinkle finishing agents reached the standard range. After glucose finishing, the WRA of the treated fabric was increased slightly, because glucose was a reducing monosaccharide, and the aldehyde group of glucose was cross-linked with the hydroxyl group of cellulose under the high temperature, which improved the WRA of the finished fabric. 


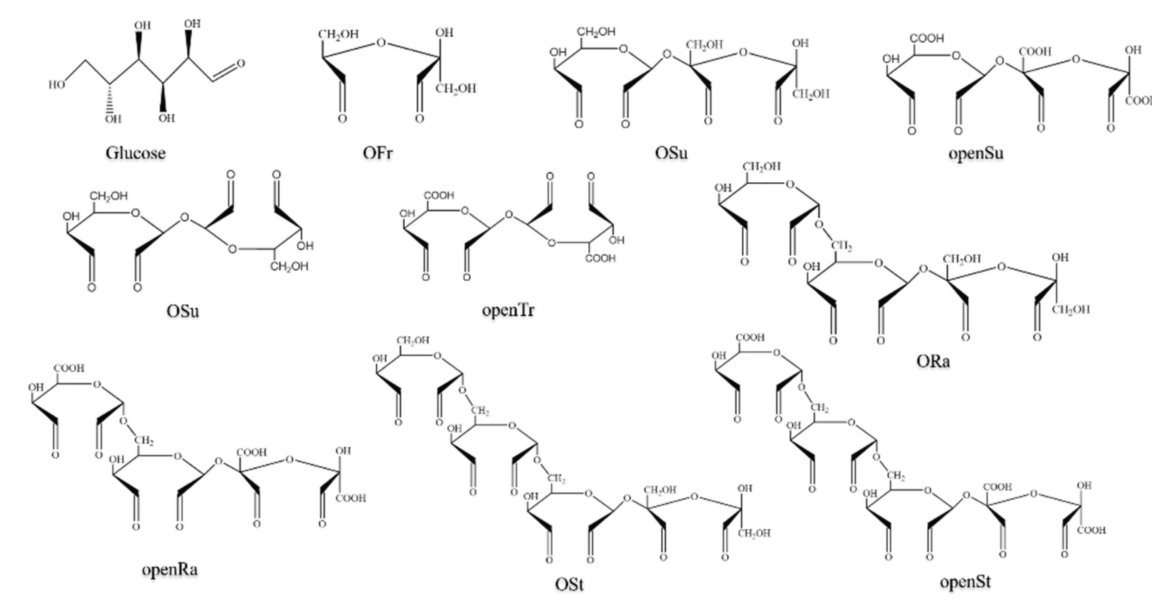

Figure 3. Structure of glycosyl polyaldehydes and glycosyl polyuronic acid finishing agents.
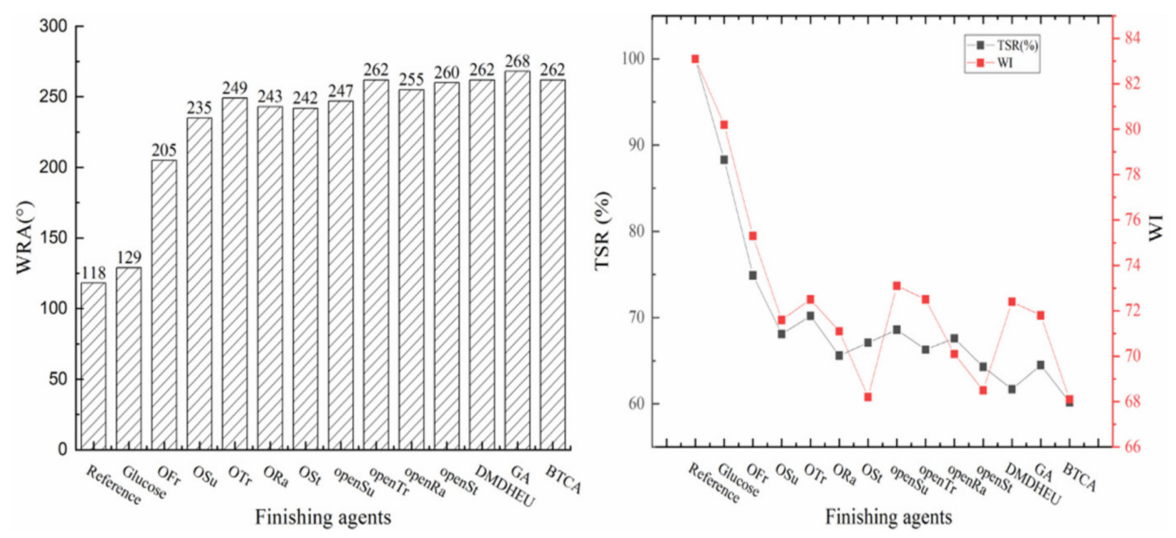

Figure 4. Comparison of anti-wrinkle performance treated with different finishing agents.

After finishing with sauger-based polyaldehyde finishing agents OSu, OTr, ORa, and OSt, the WRA of the fabric was significantly improved, and the ORa finished fabric had the highest WRA of $249^{\circ}$. The Ofr-, Osu-, Otr-, Ora-, Ost-finished fabrics had large differences in TS and WI, the WRA of the OFr-finished fabric was the best and the WRA of the OStfinished fabric was the worst. It may be because OFr has a low aldehyde content, while OSt has a higher aldehyde group content than OFr. The aldehyde group fully reacted with the cellulose hydroxyl group during the high-temperature curing, leading the strength and whiteness of the fabric to be significantly reduced.

Compared with the fabrics finished with glyco-polyaldehyde finishing agents, the WRAs of the fabrics finished with openSu, openTr, openRa, and openSt were higher; the WRA of the openTr-finished fabric reached $262^{\circ}$. This was because the molecular structure of openTr was relatively symmetrical, and the molecular volume was small. The openSu-, openTr-, openRa-, and openSt-finished fabrics have good TS, which were above $68 \%$, and the WI of the finished fabrics was around 70.

\subsection{Molecular Size Analysis of Glycosyl Anti-Wrinkle Finishing Agent}

The anti-wrinkle finishing agent has a difference in molecular size due to the mutual attraction and repulsion between the functional groups in the molecular structure. The molecular size of the finishing agent determined the diffusion speed in solution, the adsorption performance of the cellulose, and the diffusion speed into the fiber [17-19,21]. Therefore, we calculated the CSEV of OFr, OSu, OTr, openSu, openTr, and BTCA, GA, and DMDHEU according to the method in Section 2.2.5. We calculated the spherical model radius of different finishing agent molecules according to formula one (due to the 
larger molecular structure of raffinose and stachyose, the molecules cannot be regarded as spherical at the lowest energy; therefore, they have not been analyzed), and the CSEV of different anti-wrinkle agents are shown in Figure 5.

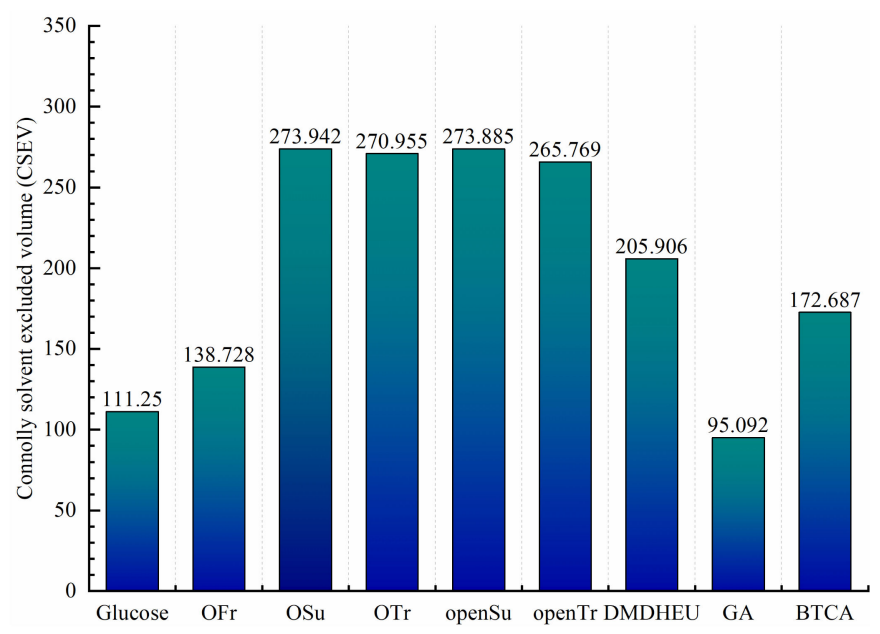

Figure 5. CSEV of different anti-wrinkle finishing agents.

As shown in Figure 5, the CSEV values of glucose and OFr were 111.25 and 138.728, respectively. The CSEV of monosaccharides was similar to the CSEV of GA, and their molecular radius was smaller, which made it easier to diffuse into the fiber. However, the structure of monosaccharides has fewer reactive groups and cannot form more effective cross-links with cotton fibers. The wrinkle resistance of the finished fabric was poor. As an example, compared with GA, OFr also contained two aldehyde groups, but the aldehyde groups in its structure were prone to condensation, which reduced the content of aldehyde groups of OFr. Therefore, the anti-wrinkle properties of OFr- and GA-finished fabrics were different.

The CSEV difference between OSu and openSu was relatively small, 273.942 and 273.885 , respectively, indicated that their diffusion performance was similar in the cotton fabrics, but the wrinkle resistance performance of their finished fabrics was different, which was related to the higher aldehyde group content of openSu and had the carboxyl groups in their structure. The same CSEV difference between OTr and openTr was also small, 265.769 and 270.955, respectively, but the wrinkle resistance of their finished fabrics was also quite different. The molecular sizes of ORa, OSt, openRa, and openSt, which cannot be regarded as spherical, were much larger than that of disaccharide anti-wrinkle finishing agents, which made them difficult to diffuse into the cellulose and form effective cross-linking with cellulose. Therefore, the anti-wrinkle performance of their finished fabric was poor.

It was speculated that although the molecular radius of the finishing agent affected its absorption and diffusion into the cellulose, the final anti-wrinkle performance of the finished fabric was mainly determined by the cross-linking performance and the type and number of reactive groups of the anti-wrinkle finishing agent.

\subsection{Cross-Linking Performance of Glycosyl Anti-Wrinkle Finishing Agent}

The anti-wrinkle finishing agent will react with cellulose from being absorbed onto the surface of the fiber and being diffused into the fiber. The degree of crosslinking was mainly affected by the type and number of reactive groups of the finishing agent molecule $[15,16,22,23]$. Therefore, by analyzing the influencing factors of the aldol reaction and the esterification reaction between the finishing agent and cellulose, the number of reactive groups and rotatable bonds of the finishing agent, the cross-linking performance of glucose, $\mathrm{OFr}, \mathrm{OSu}, \mathrm{OTr}$, openSu, openTr, BTCA, GA, and DMDHEU can been analyzed. The molecular structures of different finishing agents at the lowest energy are shown in 
Figure 6, and the number of reactive groups and rotatable bonds of the finishing agent are shown in Table 1.

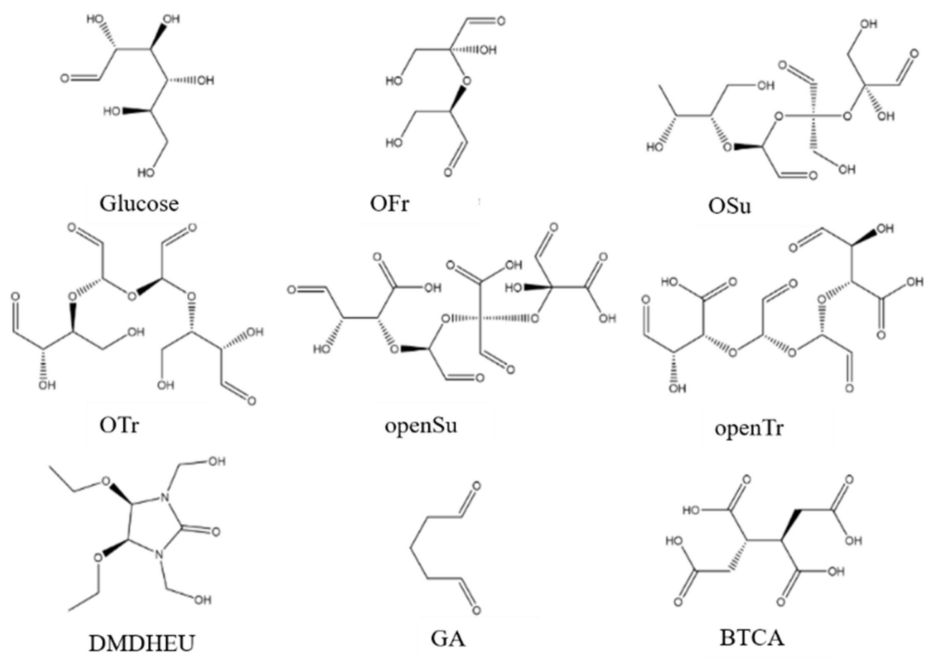

Figure 6. Molecular structure of different finishing agents at the minimum energy.

Table 1. Comparison of cross-linking properties of different finishing agent molecules.

\begin{tabular}{ccccc}
\hline Finishing Agents & Molecular Weight & PCG & NRS & RBN Value \\
\hline Glucose & 180.156 & 1 & 2 & 5 \\
OFr & 178.140 & 2 & 4 & 6 \\
OSu & 354.264 & 4 & 8 & 14 \\
OTr & 338.265 & 4 & 8 & 14 \\
openSu & 396.213 & 7 & 9,10 & 14 \\
openTr & 366.231 & 6 & 9 & 14 \\
DMDHEU & 324.252 & 2 & 2 & 6 \\
GA & 100.117 & 2 & 4 & 4 \\
BTCA & 234.160 & 4 & 2,3 & 7 \\
\hline
\end{tabular}

In Figure 6, there are mainly two types of reactive groups (carboxyl group and aldehyde group) of the nine anti-wrinkle finishing agents. According to the esterification reaction mechanism in polycarboxylic acids, more than two carboxyl groups are required to be dehydrated into an acid anhydride, which can react with the hydroxyl group of cellulose [2,5-7]. Therefore, a single carboxyl group cannot be used as a reactive group. As to BTCA, there were four carboxyl groups in the molecule; according to the reaction mechanism of the two-by-two acid anhydride formation between the carboxyl groups, the maximum number of carboxyl groups that can form an esterification reaction was three, but there were two trans- $\mathrm{COOH}$ of BTCA, which made it difficult to form an anhydride; therefore, the number of reactive groups of BTCA was four, and the number of reaction sites was two or three. According to the aldol etherification reaction mechanism, after the aldehyde group reacted with the hydroxyl group to form an ether bond, this aldehyde group formed a highly reactive hydroxyl group, which can also continue to react with the hydroxyl group of cellulose; therefore, an aldehyde group will react with two hydroxyl groups. Using glutaraldehyde (GA) as an example, there were two aldehyde groups, assuming the aldehyde groups can fully react with the hydroxyl groups of cellulose to form four ether bonds $[10,11]$. Therefore, the number of reactive groups of GA was two and the number of reaction sites was four. The reactive group of DMDHEU was N-hydroxymethyl, which formed an ether bond with the hydroxyl group of cellulose [1,3,4]. One N-hydroxymethyl can only form one ether bond; therefore, the number of reactive groups of DMDHEU was two and the number of reaction sites was two. 
Through these three calculation methods, the number of reactive groups and the number of reaction sites of the nine anti-wrinkle finishing agents are summarized in Table 1, and the number of rotatable bonds (RBN value) of the different finishing agents are also summarized. The RBN value can be used to analyze the flexibility of the finishing agent molecule, which can indicate the possibility of the finishing agent colliding with the hydroxyl group of the cellulose. The larger the RBN value is, the more likely the finishing agent molecules is to rotate, and the cross-linking efficiency of the finishing agent and cellulose were also higher.

Comparing glucose and $\mathrm{OFr}$, there were two aldehyde groups in the molecular structure of $\mathrm{OFr}$, which had more cross-linking sites with cellulose. Therefore, the degree of cross-linking was higher, and the OFr-finished cotton fabric had higher WRA. However, the number of aldehyde groups of OFr was far less than that of the disaccharide finishing agents. Among them, OSu and OTr had four reactive groups, four reactive sites, and the RBN value was 14, which indicated that OSu and OTr are more likely to undergo crosslinking reactions with cellulose. Although the cross-linking performances of OSu and OTr were similar, OTr had the smaller molecular weight and molecular radius (Figure 4), which was easier to diffuse into the cellulose, and the degree of cross-linking and the anti-wrinkle property of the finished fabrics was higher. However, the molecules of OSu and OTr were prone to self-polymerization, and the molecular weight and molecular radius were also larger. Compared with GA, the anti-wrinkle performance of their finished fabrics was worse than that of the GA-finished fabrics.

The number of reactive groups of openSu and openTr were seven and six, the number of reactive sites were 9 or 10 and 9, respectively, and the RBN value was 14 . Our previous research proved that the aldehyde and carboxyl groups can be cross-linked with the hydroxyl group of cellulose under a mixed catalyst and two-step curing conditions, but the anti-wrinkle performance of the openSu- and openTr-finished fabrics were different. The possible reasons were that the molecular structure of openSu was asymmetrical compared with openTr, the internal steric hindrance of the molecule was larger, and the three carboxyl groups in the molecular structure had greater repulsion. Therefore, although openSu had more reactive groups and the cross-linking sites were relatively small, the molecular structure of openTr was symmetrical and the molecular radius was small, which was easier to diffuse inside the cellulose to form the cross-linked network.

\subsection{Effective Crosslinking Radius of Glycosyl Anti-Wrinkle Finishing Agent}

According to the chemical cross-linking anti-wrinkle finishing mechanism of cotton fabrics, the finishing agent has at least two cross-linking reactive groups; therefore, the cross-linking agent can form effective cross-links with cellulose. After the finishing agent molecules were adsorbed and diffused into the fiber, one reactive group was cross-linked with cellulose and the remaining reactive groups needed to be cross-linked with cellulose to be counted as effective cross-linking [8,9,23-25]. Therefore, we combined one reactive group of the anti-wrinkle finishing agent with the hydroxyl group of the cellulose, calculated the rotation of the finishing agent molecule itself to form its effective crosslinking radius, and analyzed the effective crosslinking radius inside the cellulose.

To analyze the effective crosslinking radius of BTCA, GA, OTr, and openTr, Figure 7 shows the molecular structures of the four finishing agents after one single reactive group was combined with cellulose. Through the method in Section 2.2.7, the energy probability distribution of the cross-linking agent under different cross-linking conditions was calculated, and the results are shown in Table 2. 


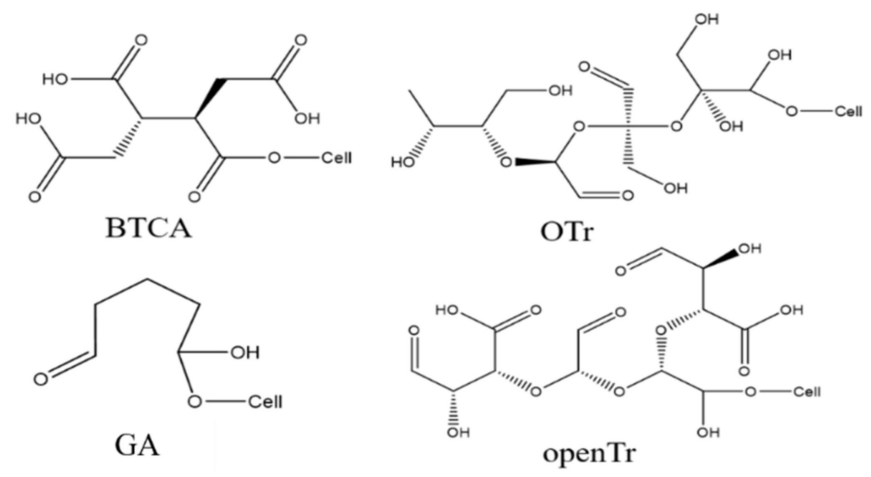

Figure 7. Single crosslinking between cellulose and crosslinker.

Table 2. Energy distribution ratio under different conformations with single cross-linked.

\begin{tabular}{cccc}
\hline Molecular Conformations & Molecular Conformational Energy (a.u.) & $\delta$ E (kcal/mol) & Proportion \\
\hline BTCA-1 & -911.72876 & 0 & $96.87 \%$ \\
BTCA-2 & -911.724877 & 2.44 & $1.58 \%$ \\
BTCA-3 & -911.724415 & 2.73 & $0.97 \%$ \\
BTCA-4 & -911.723258 & 3.45 & $0.28 \%$ \\
BTCA-5 & -911.722893 & 3.68 & $0.19 \%$ \\
BTCA-6 & -911.721756 & 4.4 & $0.06 \%$ \\
BTCA-7 & -911.721025 & 4.85 & $0.03 \%$ \\
BTCA-8 & -911.720344 & 5.28 & $0.01 \%$ \\
GA-1 & -345.36023 & 0 & $88.56 \%$ \\
GA-2 & -345.358668 & $0.46 \%$ \\
GA-3 & -345.356575 & 0.98 & $0.92 \%$ \\
GA-4 & -345.35652 & 2.29 & $0.87 \%$ \\
GA-5 & -345.355609 & 2.33 & $0.66 \%$ \\
GA-6 & -345.355221 & 2.9 & $0.44 \%$ \\
GA-7 & -345.354292 & 3.14 & $0.08 \%$ \\
OTr-1 & -1294.015689 & 3.73 & $74.23 \%$ \\
OTr-2 & -1294.014191 & 0 & $15.17 \%$ \\
OTr-3 & -1294.01368 & 0.94 & $8.83 \%$ \\
OTr-4 & -1294.011451 & 1.26 & $0.83 \%$ \\
OTr-5 & -1294.010956 & 2.66 & $0.49 \%$ \\
OTr-6 & -1294.01078 & 2.97 & $0.41 \%$ \\
OTr-7 & -1294.008548 & 3.08 & $0.04 \%$ \\
openTr-1 & -1441.946577 & 4.48 & $02.31 \%$ \\
openTr-2 & -1441.944137 & $0.95 \%$ \\
openTr-3 & -1441.941999 & 1.53 & $6.72 \%$ \\
openTr-4 & -1441.938257 & 2.87 & $0.01 \%$ \\
\hline & & 5.22 & \\
\hline
\end{tabular}

It can be seen from Table 2 that OTr can form seven different configurations through its own rotation, which shows that $\mathrm{OTr}$ has seven possible molecular structures inside cellulose, of which the largest proportion and the lowest energy conformation accounts for $74.23 \%$. The relatively symmetrical molecular structure of openTr has only four lower energy conformations, and the lowest energy conformation accounts for $92.31 \%$, while BTCA and GA have eight conformations and seven conformations, and the lower energy conformation proportions are 96.87 and $88.56 \%$, respectively. The distance from the reaction site to the assumed cross-linking point under the corresponding cross-linking was measured using Chem-craft software (Figure 8), the distances that can form cross-links in different conformations were summarized. We used the cross-linking distance as the abscissa and the fraction as the ordinate to draw a fan-shaped distribution diagram, and the result is shown in Figure 9. 


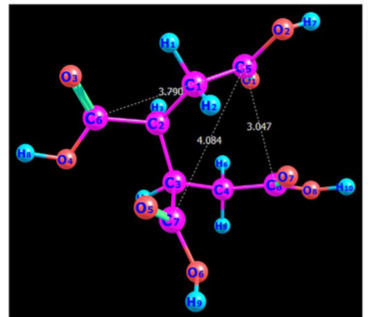

BTCA-1

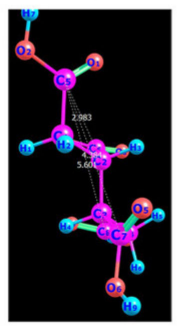

BTCA-5

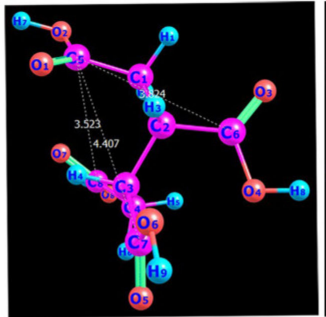

BTCA-2

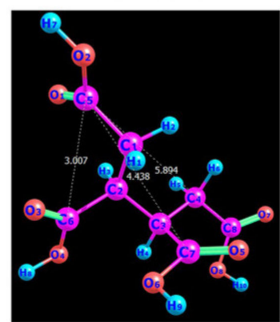

BTCA-6

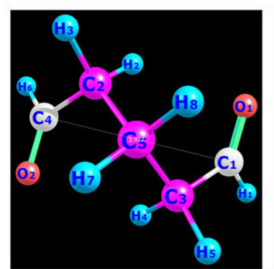

GA-1

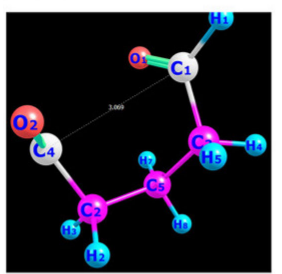

GA-5

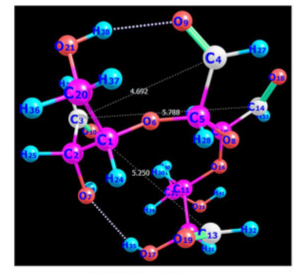

OTr-1

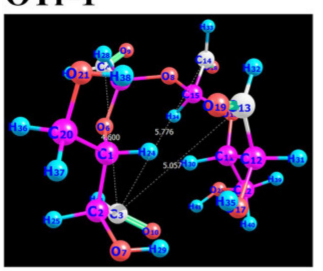

OTr-5

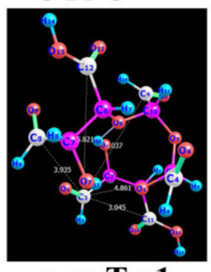

openTr-1

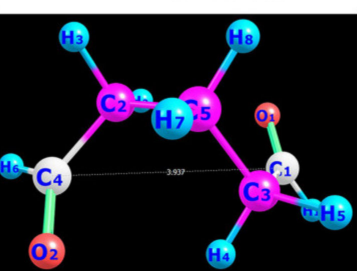

GA-2

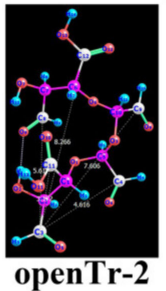

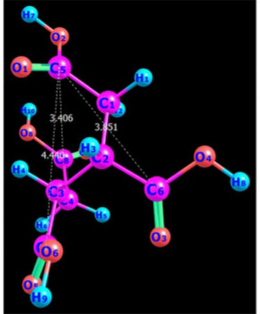

BTCA-3

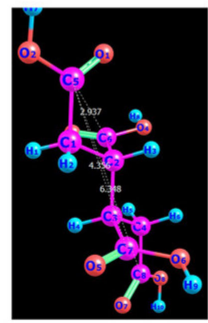

BTCA-7

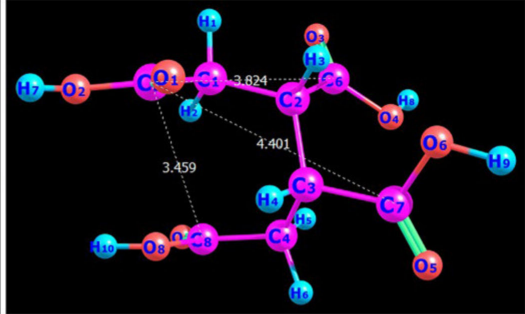

BTCA-4

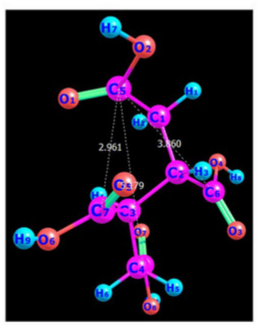

BTCA-8

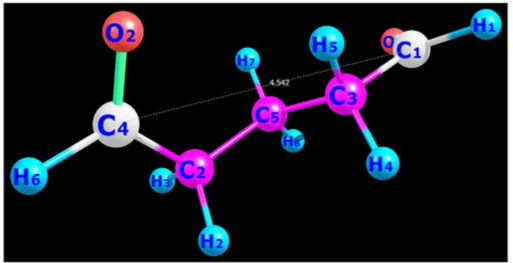

GA-3

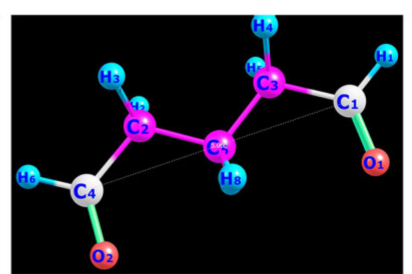

GA-4

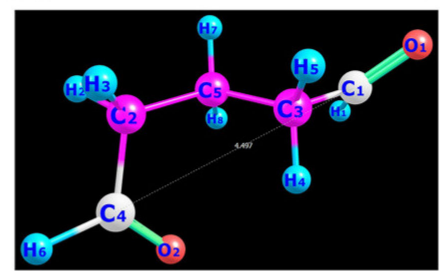

GA-6

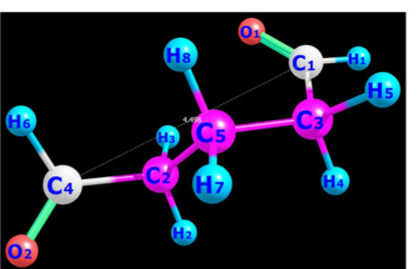

GA-7

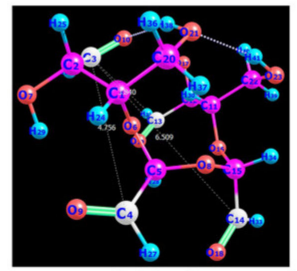

OTr-2

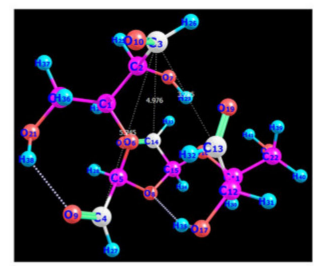

OTr-6

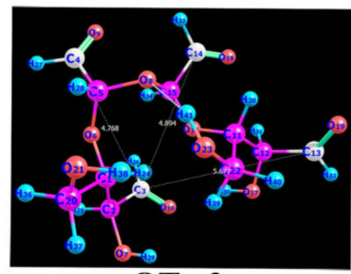

OTr-3

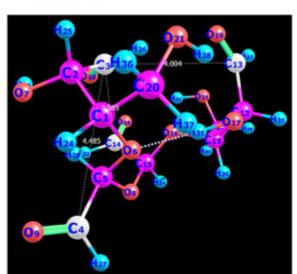

OTr-4

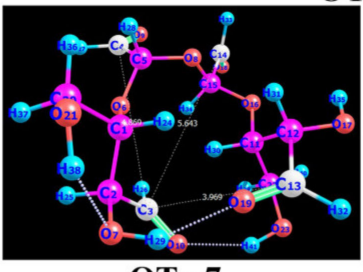

OTr-7

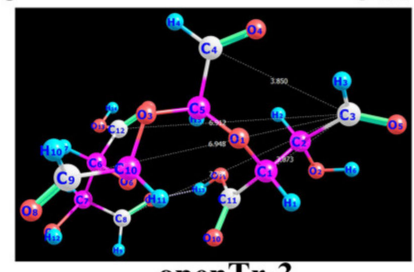

openTr-3

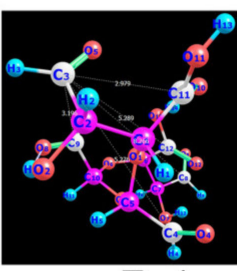

openTr-4

Figure 8. Molecular structure in different conformations and the distance between two cross-linking points. 

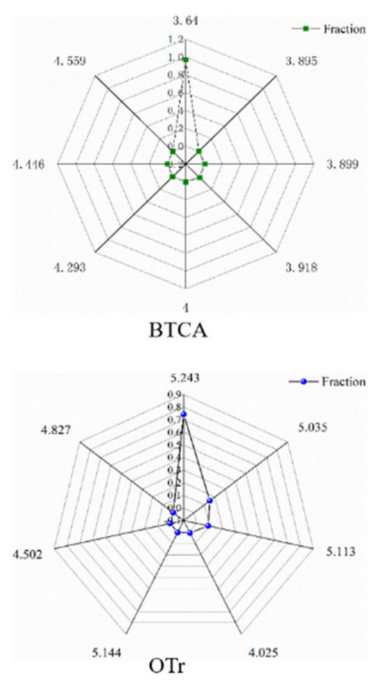

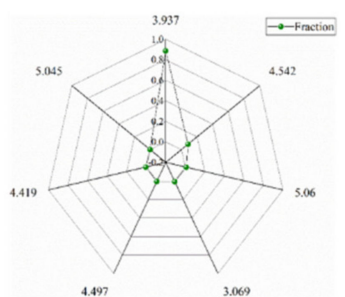

GA

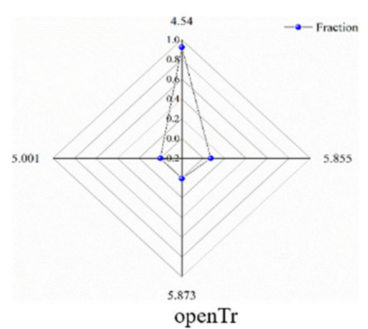

Figure 9. Fan-shaped distribution of effective cross-linking distance.

In Figure 9, the cross-linking distance with the highest proportion among BTCA of different conformations was $3.64 \AA$, accounting for $96.89 \%$, which was the most effective cross-linking radius range of BTCA; the lowest proportion reaches the effective distance of $4.559 \AA$, which was also the longest cross-linking range. Beyond this range, the probability of BTCA cross-linking was low; therefore, the effective cross-linking radius of BTCA was $3.64 \AA-4.559 \AA$. According to this method, the effective cross-linking radius with the highest proportion of glutaraldehyde was $3.937 \AA$, the proportion was $74.26 \%$, and its effective radius was 3.937-5.045 $\AA$. The effective cross-linking radius with the highest proportion of OTr was $5.243 \AA$, accounting for $74.23 \%$, and their effective radius was $4.025-5.045 \AA$. The highest effective cross-linking radius of openTr was $4.54 \AA$, accounting for $92.31 \%$, and its effective radius was 4.540-5.873 $\AA$.

According to the similar simulation method, we simulated other glycosyl polyaldehyde and glycosyl polyuronic acid finishing agents, calculated their effective cross-linking distribution radius, and arranged these cross-linking distributions in a pyramid shape, which is shown in Figure 10.

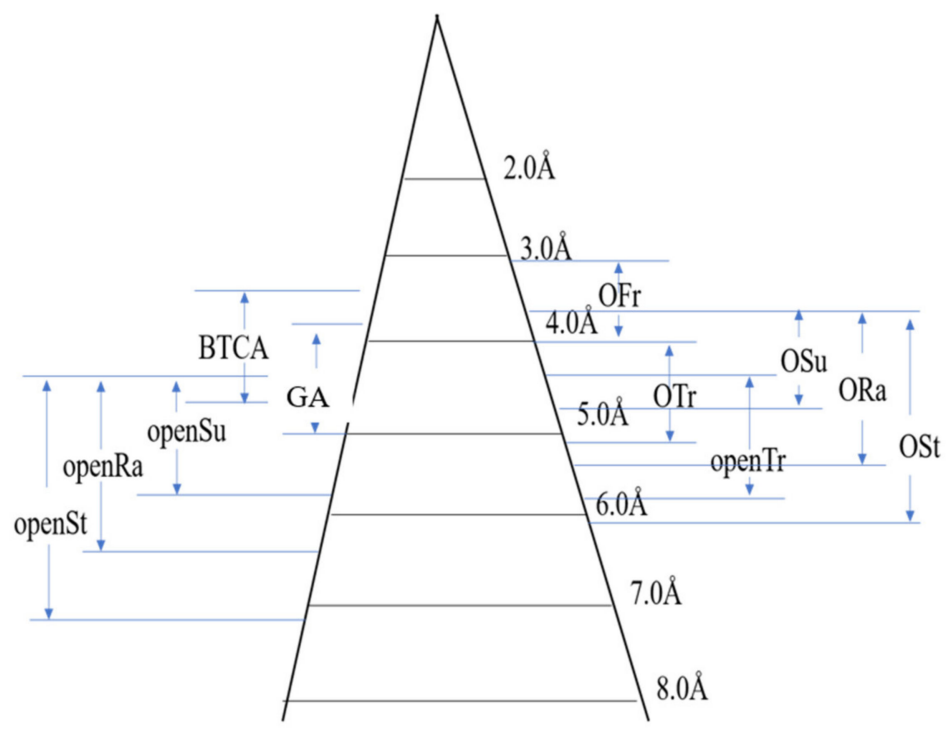

Figure 10. Effective crosslinking radius distribution of glycosyl anti-wrinkle finishing agents.

It can be seen from Figure 9 that OFr, GA, and BTCA, as finishing agents with smaller molecular weights, have a smaller effective crosslinking radius. However, the anti-wrinkle 
property of GA was better than OFr because OFr's own molecular radius was small, meaning that effective cross-linking cannot be formed in the fiber. The cross-linking radius distributions of OFr, OSu, OTr, Ora, and OSt ranged from 3.6 to $6.1 \AA$. Among them, because the radius of the OTr and ORa finishing agents are relatively small, the anti-wrinkle performances of OTr and ORa were better. When OTr and ORa entered the cellulose, they directly crosslinked with cellulose, or formed dimers or multimers, and then the number of reaction sites with cellulose was increased. Although the effective cross-linking radius of OSt was the largest, it was difficult to diffuse into the cellulose; therefore, the anti-wrinkle performance of Ost-finished fabrics was poor.

The cross-linking radius of openSu, openTr, openRa, and openSt ranged from $4.5 \AA$ to $7.3 \AA$, of which openTr had the best anti-wrinkle performance. In the case that the glycosyl polyuronic acid finishing agent could not be polymerized intramolecularly or intermolecularly, the molecular weight was small and the structure was regular, which was easier to diffuse into the cellulose; therefore, the number of cross-linking agents inside the fiber was increased, the more effective cross-linking was able to form, and the antiwrinkle performance of finished fabrics was better. Therefore, combined with the effective cross-linking radius distribution of GA and BTCA, it can be roughly speculated that the optimal cross-linking radius ranges of glycosyl polyaldehyde and glycosyl polyuronic acid finishing agent were $3.5 \AA-6.0 \AA$.

\section{Conclusions}

This paper studied the anti-wrinkle performance of glycosyl polyaldehyde finishing agents (OFr, OSu, OTr, ORa, OSt), glycosyl polyuronic acid finishing agents (openSu, openTr, openRa and openSt) on finished cotton fabrics. Among them, OTr-, Ora-, openTrand openRa- finished fabrics showed excellent an anti-wrinkle property, the TS of the finished fabric was above $68 \%$, and the WI was above 70 . The molecular radius of the glycosyl polyaldehyde finishing agents and the glycosyl polyuronic acid finishing agents were calculated using the MM2 method; the smaller the molecular volume of the finishing agent was, the easier it was to diffuse into the cotton fiber, and the higher the amount of finishing agent was inside the cotton fiber, which was conducive to the effective crosslinking of the finishing agent with the cotton fiber. The number of reactive groups, the number of reactive sites, and the RBN value of the finishing agent affected the crosslinking reaction with cellulose. The more reactive the groups and sites were, the greater the RBN value was, which was more conducive to the occurrence of the crosslinking reaction. The cross-linking radius range of glycosyl formaldehyde-free finishing agent was analyzed using a molecular simulation. The most suitable cross-linking radius range was 3.5-6.0 $\AA$; within this range, the anti-wrinkle finishing agent was easily diffused to the inside of the cellulose. This study provides a reference for the design of new formaldehyde-free anti-wrinkle finishing agents with new molecular structures.

Author Contributions: Conceptualization, X.F.; Software and writing—original draft preparation, J.L.; Writing - review and editing, D.W. and J.Y. All authors have read and agreed to the published version of the manuscript.

Funding: This work was financially supported by the National Key R\&D Program of China (2017YFB0309202) and Postgraduate Research \& Practice Innovation Program of Jiangsu Provence (1062050205198056).

Institutional Review Board Statement: Not applicable.

Informed Consent Statement: Not applicable.

Data Availability Statement: Not applicable.

Conflicts of Interest: The authors declare no conflict of interest. 


\section{References}

1. Dehabadi, V.A.; Buschmann, H.-J.; Gutmann, J.S. Durable press finishing of cotton fabrics: An overview. Text. Res. J. 2013, 83, 1974-1995. [CrossRef]

2. Harifi, T.; Montazer, M. Past, present and future prospects of cotton cross-linking: New insight into nano particles. Carbohydr. Polym. 2012, 88, 1125-1140. [CrossRef]

3. Chang, H.-L.; Chen, C.-C. Crosslinking of Cotton with DMDMDHEU in the Presence of Sodium Chloride. Text. Res. J. 2016, 66, 803-809. [CrossRef]

4. Chen, J.C.; Yao, W.H.; Chen, C.H.; Chen, C.C. Dyeing kinetics from a finite bath of direct dyes on DMDHEU-AA crosslinked cotton. Text. Res. J. 2002, 72, 55-60.

5. Lund, K.; Brelid, H. Kinetics of Cross-Linking Softwood Kraft Pulp with 1,2,3,4-Butanetetracarboxylic Acid. Ind. Eng. Chem. Res. 2013, 52, 11502-11509. [CrossRef]

6. Wang, H.; Zhang, C.; Chu, X.; Zhu, P. Mechanism of Antiwrinkle Finishing of Cotton Fabrics Using Mixed Polycarboxylic Acids. Int. J. Polym. Sci. 2020, 2020, 3876595. [CrossRef]

7. Zhang, X.; Ji, B.; Yan, K.; Hu, T. Non-phosphorus catalysts for the ester cross-linking of cellulose with 1,2,3,4-butanetetracarboxylic acid. Fibers Polym. 2017, 18, 682-688. [CrossRef]

8. Ji, B.; Zhao, C.; Yan, K.; Sun, G. Effects of acid diffusibility and affinity to cellulose on strength loss of polycarboxylic acid crosslinked fabrics. Carbohydr. Polym. 2016, 144, 282-288. [CrossRef]

9. Munster, L.; Vicha, J.; Klofac, J.; Masar, M.; Hurajova, A.; Kuritka, I. Dialdehyde cellulose crosslinked poly(vinyl alcohol) hydrogels: Influence of catalyst and crosslinker shelf life. Carbohydr. Polym. 2018, 198, 181-190. [CrossRef]

10. Liu, P.; Xu, H.; Mi, X.; Xu, L.; Yang, Y. Oxidized Sucrose: A Potent and Biocompatible Crosslinker for Three-Dimensional Fibrous Protein Scaffolds. Macromol. Mater. Eng. 2015, 300, 414-422. [CrossRef]

11. Muhammad, M.; Willems, C.; Rodriguez-Fernandez, J.; Gallego-Ferrer, G.; Groth, T. Synthesis and Characterization of Oxidized Polysaccharides for In Situ Forming Hydrogels. Biomolecules 2020, 10, 1185. [CrossRef] [PubMed]

12. Pei, J.; Yin, Y.; Shen, Z.; Bu, X.; Zhang, F. Oxidation of primary hydroxyl groups in chitooligomer by a laccase-TEMPO system and physico-chemical characterisation of oxidation products. Carbohydr. Polym. 2016, 135, 234-238. [CrossRef] [PubMed]

13. Wei, C.; Tao, W.; Lickfield, G.C. Molecular modeling of cellulose in amorphous state. III. An innovative elastomeric crosslink system. J. Polym. Sci. Part B Polym. Phys. 2010, 45, 1821-1833.

14. Chen, W.; Lickfield, G.C.; Yang, C.Q. Molecular modeling of cellulose in amorphous state. Part I: Model building and plastic deformation study. Polymer 2004, 45, 1063-1071. [CrossRef]

15. Wu, C.; Xu, W. Atomistic molecular modelling of crosslinked epoxy resin. Polymer 2006, 47, 6004-6009. [CrossRef]

16. Yarovsky, I.; Evans, E. Computer simulation of structure and properties of crosslinked polymers: Application to epoxy resins. Polymer 2002, 43, 963-969. [CrossRef]

17. Yo, A.; Mn, A.; Ks, A.; Yh, A.; Ki, A.; Gk, B.; Jing, L.A.; Rk, C.; Nk, D.; Hw, D. Molecular dynamics simulation of cross-linking processes and material properties for epoxy resins using first-principle calculation combined with global reaction route mapping algorithms. Chem. Phys. Lett. 2020, 762, 138104.

18. Zhang, W.; Wang, Z.; Lv, S.; Zhan, W.; Yang, X. Molecular simulation of different structure dopamine-modified graphene oxide and its effects on thermal and mechanical properties of the epoxy resin system. Polymer 2020, 212, 123120. [CrossRef]

19. Hai, B.F.; Yuen, M. Material properties of the cross-linked epoxy resin compound predicted by molecular dynamics simulation. Polymer 2007, 48, 2174-2178.

20. Nan, T.; Ning, R.; Jie, K. Self-toughening of epoxy resin through controlling topology of cross-linked networks. Polymer 2016, 99, 376-385.

21. Hsiung, H.H.; Huang, H.Y.; Wang, Y.H.; Wang, C.; Chen, C.C. Physical properties and reaction kinetics of cotton cellulose crosslinked with dimethyloldihydroxyethyleneurea-maleic acid. J. Appl. Polym. Sci. 2010, 92, 3886-3893. [CrossRef]

22. Park, C.; Kim, G.; Jung, J.; Krishnakumar, B.; Ranac, S.; Yun, G.J. Enhanced self-healing performance of graphene oxide/vitrimer nanocomposites: A molecular dynamics simulations study. Polymer 2020, 206, 122862. [CrossRef]

23. Madan, G.L.; Patel, S.B.; Baddi, N.T.; Mehta, P.C. Physical Chemistry of Crosslinking: Part I: Thermodynamics of Cellulose in Aqueous Solution of Crosslinking Agents. Text. Res. J. 2016, 46, 329-342. [CrossRef]

24. Ziifle, H.M.; Benerito, R.R.; Gonzales, E.J.; Berni, R.J. Kinetics of the Reactions of Ethyleneurea Derivatives with Cotton Cellulose. Text. Res. J. 2016, 38, 925-930. [CrossRef]

25. Dang, X.; Liu, P.; Yang, M.; Deng, H.; Shan, Z.; Zhen, W. Production and characterization of dialdehyde cellulose through green and sustainable approach. Cellulose 2019, 26, 9503-9515. [CrossRef] 